\title{
Supernova neutrino physics in future water-Cherenkov detectors
}

\author{
Gianluigi Fogli \\ Dip.to di Fisica and Sezione INFN, Bari, Italy \\ E-mail: gianluigi.fogli@ba.infn.it
}

Eligio Lisi*

Sezione INFN, Bari, Italy

E-mail: eligio.lisi@ba.infn.it

\section{Alessandro Mirizzi}

Dip.to di Fisica and Sezione INFN, Bari, Italy

E-mail: alessandro.mirizzi@ba.infn.it

\section{Daniele Montanino}

Dip.to di Scienza dei Materiali and Sezione INFN, Lecce, Italy

E-mail: daniele.montanino@unile.it

\begin{abstract}
We study the physics potential of a reference next-generation water-Cherenkov detector ( 0.4 Mton of fiducial mass) in providing information on supernova neutrino flavor transitions with unprecedented statistics. We emphasize the importance of studying the time spectra of events, which encode combined information on neutrino oscillation parameters and on supernova forward (and possibly reverse) shock waves. In particular, we show that an appropriate ratio of low-to-high energy events can faithfully monitor the time evolution of the neutrino crossing probability along the shock-wave profile, and can provide precious information about currently unknown neutrino mass-mixing parameters.
\end{abstract}

International Europhysics Conference on High Energy Physics

July 21st - 27th 2005

Lisboa, Portugal

\footnotetext{
*Speaker.
} 


\section{Introduction}

The successful operation of the Super-Kamiokande water-Cherenkov experiment [1, 2] have motived several research groups to investigate in detail the feasibility of a Megaton-class detector [3] for nucleon decay and neutrino physics. Three main projects are currently being pursued: the Underground nucleon decay and Neutrino Observatory (UNO) project in the U.S. [4, 5], the HyperKamiokande (HK) project in Japan [6], and the MEgaton class PHYSics (MEMPHYS) project in Europe [7]. These next-generation detectors are characterized by a prospective fiducial mass of 0.4 Mton or higher, which will allow studies of neutrinos of astrophysical or terrestrial origin with unprecedented statistics and sensitivity [8]. In particular, a new window would be opened on supernova ( $\mathrm{SN}$ ) neutrino physics: by naively rescaling the detector mass, it turns out that a 0.4 Mton experiment would observe a SN1987A-like signal with a statistics $\sim 200$ times higher than in Kamiokande (2.14 kton) [3]. In general, the observation of a large number of supernova neutrino events will allow statistically significant spectral analyses in the energy, angular, and time domain.

The identification of relatively model-independent spectral features will be important to disentangle, as far as possible, information related to supernova physics and neutrino emission from those related to neutrino flavor transitions. In this context, we briefly discuss a recent topic of particular interest: the possible observation of shock-wave signatures in neutrino event spectra (via matter effects), as first emphasized in [9]. In particular, we analyze the imprint of the shock wave on the time spectra of inverse beta decay events, and show that the ratio between the number of events in two suitably chosen energy ranges can actually monitor the time dependence of the neutrino crossing probability, thus opening a unique opportunity to study shock-induced flavor transitions in "real time." The reader is referred to [10] for a more complete and technical discussion of the results. Other recent works on this topic can also be found in $[11,12,13]$.

\section{Shock waves and their observable effects on neutrinos}

In a seminal paper [9], Schirato and Fuller noted that the propagation of a forward shock wave in supernova simulations could influence neutrino flavor transitions a few seconds after core bounce. The main features of the forward shock profile are a sharp discontinuity at the shock front (which can induce a strongly nonadiabatic transition) leaving behind an extended rarefaction zone. In addition to forward shock effects, it has been recently pointed out [11] that a second ("reverse") shock front, is also expected to propagate behind the forward one (at a lower velocity), although a detailed description is not yet possible within current numerical experiments.

The effects of the time-dependent matter profile (encountered by neutrinos along their propagation through the supernova shock wave) can be conveniently embedded in a the so-called "crossing probability" $P_{H}$ which, in general, is a function of both time and energy, as well as of the (yet unknown) neutrino mixing angle $\theta_{13}$. This function can be quite complicated in the presence of forward (+reverse) shock, see [10,11] and references therein. Despite its complexity, the time dependence of $P_{H}$ can be monitored surprisingly well in future water-Cherenkov detectors. Indeed, we have shown in [10] that the function $\left[1-\cos ^{2} \theta_{12} P_{H}\right]^{-1}$ can be monitored in the time domain, by taking the ratio of inverse-beta-decay events in two properly chosen energy bins. The bin energy ranges, for the adopted supernova emission model, correspond to $E_{L}=20 \pm 5 \mathrm{MeV}$ bin and 


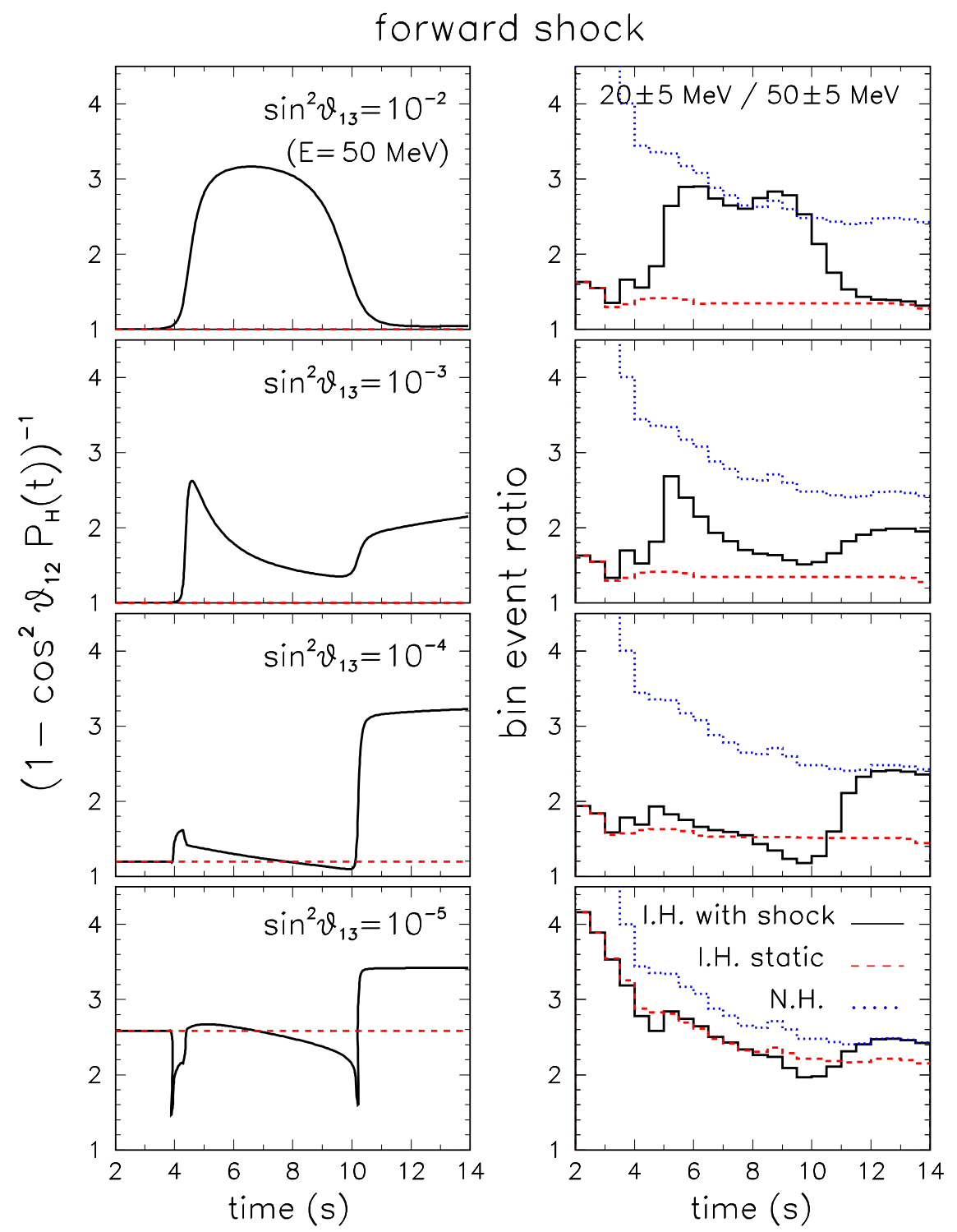

Figure 1: Time dependence of the ratio of events between the energy bins $E_{\mathrm{pos}}=20 \pm 5 \mathrm{MeV}$ and $E_{\mathrm{pos}}=50 \pm 5 \mathrm{MeV}$ (right), compared with the function $\left[1-\cos ^{2} \theta_{12} P_{H}\left(E_{H}, t\right)\right]^{-1}$ with $E_{H}=50 \mathrm{MeV}$ (right), for various values of $\sin ^{2} \theta_{13}$. It appears that, for inverted hierarchy $(\mathrm{IH})$, the event ratio on the right panels tracks rather well the function structures on the left panels (up to smearing effects), thus providing a "shock wave monitor" in real time. The effect is not seen if the hierarchy is normal $(\mathrm{NH})$ and, of course, if there is no shock wave at all (static profile). Notice that the various cases become experimentally indistinguishable for small values of $\theta_{13}$.

$E_{H}=50 \pm 5 \mathrm{MeV}$; in both of them, a galactic supernova explosion will deposit a high number of events (for a 0.4 Mton detector), so that the statistical error is not an issue in this case.

Figure 1 shows the ratio of events in the $20 \pm 5 \mathrm{MeV}$ bin with respect to events in the $50 \pm$ $5 \mathrm{MeV}$ bin, as compared with the function $\left[1-\cos ^{2} \theta_{12} P_{H}\left(E_{H}, t\right)\right]^{-1}$, for the case of forward shock propagation. [For forward+reverse shock (not shown) the spectral ratio would have an even richer structure]. This function has the same qualitative behavior as $P_{H}\left(E_{H}, t\right)$ itself, see [10]. It can be seen that, in inverted hierarchy, shock effects are faithfully reproduced by the chosen ratio of events, i.e., there is a striking correspondence (even in absolute values, within a factor of 2 or better) 
between the solid histograms on the right and the solid curves on the left in Fig. 1, up to smearing effects due to the detector resolution. For inverted neutrino mass hierarchy (IH) with no shock (dashed curves and histograms) there is also a reasonable correspondence of the event ratio on the right with the constant value on the left. For normal neutrino mass hierarchy $(\mathrm{NH})$, as expected on theoretical grounds, any information on $P_{H}$ is lost. Therefore, in the case of inverted hierarchy, the ratio of events at "high energy" and "critical energy" appears as a useful tool to track the main variations (and possibly the absolute value) of the crossing probability $P_{H}$, from which one could get precious information about the density gradient along the shock profile. In conclusion, the observation of a "wavy" pattern in the spectral ratio of Fig. 1 would be indicative of: (1) existence of shock waves on a timescale of a few seconds; (2) nonzero $\theta_{13}$; and (3) inverted neutrino mass hierarchy.

\section{Conclusions}

Megaton-class detectors will collect huge neutrino events statistics from a future galactic supernova explosion. Spectral studies will then be possible in the energy and time domain. We have shown how appropriate ratios of time spectra can monitor the expected explosion shock wave(s) in real time, and provide information about two currently unknown neutrino observables: the mixing angle between the first and third generation $\left(\theta_{13}\right)$ and the mass hierarchy (normal or inverted). A full account of these results can be found in [10].

\section{References}

[1] M. Koshiba, "Birth of neutrino astrophysics," Rev. Mod. Phys. 75, 1011 (2003).

[2] Super-Kamiokande Collaboration, Y. Fukuda et al., Nucl. Instr. Meth. A 501, 418 (2003)

[3] M. Koshiba, Phys. Rept. 220, 229 (1992).

[4] UNO official homepage: ale.physics.sunysb.edu

[5] C.K. Jung, hep-ex/0005046.

[6] K. Nakamura, Int. J. Mod. Phys. A 18, 4053 (2003).

[7] L. Mosca, talk at the Villars CERN/SPSC Meeting (Villars, Switzerland, 2004), available at nuspp.in2p3.fr/Frejus

[8] C.K. Jung, talk at Neutrino 2004, 21 st International Conference on Neutrino Physics and Astrophysics. Website: neutrino2004.in2p3.fr

[9] R.C. Schirato and G.M. Fuller, astro-ph/0205390. This paper used shock-wave simulations by J.R. Wilson and H.E. Dahled.

[10] G. L. Fogli, E. Lisi, A. Mirizzi and D. Montanino, JCAP 0504, 002 (2005).

[11] R. Tomàs, M. Kachelrieß, G. Raffelt, A. Dighe, H.T. Janka, and L. Scheck, JCAP 0409, 015 (2004).

[12] V. Barger, P. Huber and D. Marfatia, Phys. Lett. B 617, 167 (2005).

[13] B. Dasgupta and A. Dighe, arXiv:hep-ph/0510219. 